\title{
Identification and characterization of antifungal compounds from a Burkholderia strain against plant pathogenic fungi
}

\author{
Theodorus Eko Pramudito1,2, Delia Agustina1, Widyah Budinarta1, Thi Kim Ngan Nguyen ${ }^{3}$, Cahya Prihatna ${ }^{1 \star}$ and \\ Antonius Suwanto ${ }^{1,4}$
}

${ }^{1}$ Research and Development for Biotechnology, PT Wilmar Benih Indonesia, Bekasi, Jawa Barat 17530, Indonesia.

${ }^{2}$ Faculty of Biotechnology, Universitas Katolik Indonesia Atma Jaya, Cisauk, Tangerang, Banten 15345, Indonesia.

3Wilmar Innovation Center 1, 01 Research Link, 117604, Singapore.

${ }^{4}$ Department of Biology, Faculty of Mathematics and Natural Sciences, Bogor Agricultural University. Bogor, Jawa Barat, 16680, Indonesia.

Email: cahya.prihatna@id.wilmar-intl.com

Received 10 July 2019; Received in revised form 2 December 2019; Accepted 17 February 2020

\begin{abstract}
Aims: Biocontrol of fungal plant pathogens using beneficial microorganisms is a safer alternative over synthetic fungicides. PHP12 is a bacterial strain isolated from healthy oil palm rhizosphere and is closely related to the recently described Burkholderia stagnalis, a member of the Burkholderia cepacia complex. This study aimed to characterize the antifungal activity spectrum of PHP12 and identify the antifungal compounds produced by the strain.

Methodology and results: The antifungal activity of PHP12 was characterized by growing fungal strains in the presence and absence of PHP12 and measuring the radius of the antifungal zone. PHP12 inhibited the growth of fungal pathogens including Ganoderma boninense, Curvularia oryzae, Phellinus noxius and Colletotrichum capsici. However, PHP12 did not inhibit the growth of Trichoderma asperellum, a known fungal biocontrol agent. The antifungal compounds of PHP12 were precipitated using ammonium sulfate and further purified with HPLC followed by identification using Liquid Chromatography Electrospray Ionization Tandem Mass Spectrometric (LC/ESI-MS). The LC/ESI-MS analysis showed the presence of an oligopeptide with a molecular weight of $1210.63 \mathrm{Da}$. The peptide consists of heavily modified amino acids that are linked by a hexose residue.

Conclusion, significance and impact of study: Although characteristics of the antifungal compounds are similar to other antifungal peptides from Burkholderia such as occidiofungin, there have been no reports of antifungal peptides from $B$. stagnalis with the corresponding molecular weight or fragmentation profile. The novelty of the compound, as well as its antifungal spectrum, makes PHP12 an interesting strain to be investigated further as a biocontrol agent.
\end{abstract}

Keywords: Antifungal compounds, biocontrol, Burkholderia stagnalis, mass spectrometry, plant pathogen

\section{INTRODUCTION}

With more than 8,000 species of fungi known to cause plant diseases, fungal diseases greatly impact overall plant production and therefore reduction of food sources and financial income. Several plant pathogenic fungi such as Ganoderma boninense, Fusarium oxysporum, and Phellinus noxius are pathogens of oil palms that can kill infected trees when the palms are halfway through their economic life (Aderungboye, 1977). Curvularia sp. is a pathogenic fungal species that causes brown leaf spots in rice (Majeed et al., 2015) and causes leaf spot disease in oil palm seedlings (Omamor et al., 2007). Other fungal diseases are Sclerotium rot in melons that is caused by Sclerotium rolfsii (Darma et al., 2016), root rot in herbaceous crops caused by Pythium sp. (Agrios, 2005) and anthracnose in chili plants caused by Colletotrichum sp. (Than et al., 2008).

There has been a growing concern in public regarding the use of chemical pesticides to control these fungal pathogens. The use of synthetic fungicides such as hexaconazole creates major problems such as the emergence of fungicide-resistant fungi, accumulation of carcinogenic residues in commercial products and damage to beneficial soil microorganisms (Maznah et al., 2015). A potentially harmless alternative is the use of biocontrol agents in the form of antagonistic microorganisms to inhibit fungal pathogens. Several fungal strains of Trichoderma have been reported to be able to inhibit fungal pathogens through antibiosis, parasitism, and competition for nutrients (Ghisalberti and Rowland, 2004). Several Bacillus species produce 
lipopeptides that act as a surfactant that disrupt the membrane of pathogenic fungi (Romero et al., 2007). The species Burkholderia cepacia has been considered as a biocontrol agent against Rhizoctonia by producing the antifungal pyrrolnitrin (Hwang et al., 2002).

In this study, we characterized a soil bacterium that is designated as isolate PHP12 that was isolated from soil in an oil palm plantation that was free from Ganoderma infestation. Soil bacteria belonging to the genera Burkholderia, Bacillus and Azotobacter can act as plantgrowth promoters and are also ideal candidates for biocontrol (Glick and Bashan, 1997). It could be due to their living environment in which they have to compete with fungi to colonize roots. In this paper, we discuss in more detail the identification of the isolate PHP12, its antifungal activity, and the elucidation of antifungal compounds produced by PHP12.

\section{MATERIALS AND METHODS}

\section{Chemicals and reagents}

All solvents and reagents were of analytical grade or the highest grade available. Ammonium sulfate and acetonitrile were purchased from Merck (Darmstadt, Germany). Trifluoroacetate (TFA) was purchased from Sigma-Aldrich (St. Louis, MO, USA). Difco ${ }^{\mathrm{TM}}$ Potato Dextrose Agar (PDA) was purchased from Becton, Dickinson, and Company (Sparks, MD, USA). Analyticalgrade water was obtained from ultrafiltration using Milli- $\mathrm{Q}^{\circledR}$ Integral Water Purification System for Ultrapure Water (Millipore; Molsheim, France).

\section{Isolation and identification of PHP12}

Isolation of PHP12 was performed based on the protocol described by Cordova-Kreylos et al. (2013). The soil sample was collected from Permata Hijau Plantation (PHP), West Sumatra, Indonesia, from a healthy oil palm rhizosphere $30 \mathrm{~cm}$ below the soil surface. The PHP12 isolate was maintained on King's $\mathrm{B}$ agar at $30{ }^{\circ} \mathrm{C}$ or in Luria broth (LB) medium with $20 \%(\mathrm{v} / \mathrm{v})$ glycerol at $-80{ }^{\circ} \mathrm{C}$ for long term preservation.

The PHP12 isolate was identified by sequencing the 16S rRNA gene using the 27F (5'GAGTTTGATCCTGGCTCAG-3') and 1387R (3'GAGTTTGATCCTGGCTCAG-5') primers (Macrogen, Korea). The gene sequencing was carried out using the ABI 3130 Genetic Analyzer (Thermo Scientific). Identification of strain PHP12 was conducted using nucleotide Basic Local Alignment Search Tool (BLASTn; https://blast.ncbi.nlm.nih.gov/Blast.cgi). To determine the evolutionary relationship of PHP12 within the Burkholderia, 16S rRNA sequences from 23 different Burkholderia species were retrieved from National Center for Biotechnology Information (https://www.ncbi.nlm.nih.gov/). 16S rRNA sequence of Ralstonia solanacearum was used as an outgroup. Sequences were aligned using ClustalW in Mega $X$ software (Kumar et al., 2018). The phylogenetic tree was constructed using the Neighbor-Joining method. Tree topology was inferred with the Tamura-Nei correction model (Tamura and Nei, 1993) with 1,000 bootstrap replications based on the best substitution model.

\section{In vitro antifungal assay of PHP12 against several species of fungi}

A single colony of PHP12 was streaked over King's B agar and incubated for $48 \mathrm{~h}$ at $30^{\circ} \mathrm{C}$. Single colonies were then transferred to $10 \mathrm{~mL}$ of King's B liquid media and incubated overnight. After incubation period was complete, PHP12 culture was adjusted to OD 0.5 at 600 $\mathrm{nm}$, and $100 \mu \mathrm{L}$ of culture was inoculated into $50 \mathrm{~mL}$ King's B broth followed by $24 \mathrm{~h}$ incubation at $30^{\circ} \mathrm{C}$.

Antifungal activity of PHP12 was tested using the agar well diffusion assay that is modified from the dual culture assay described by Katoch and Pull (2017). The overnight PHP12 culture was centrifuged at $10,000 \times g$ for 2 min, and the supernatant was decanted and boiled for $10 \mathrm{~min}$ to deactivate the remaining microorganisms. The boiled supernatant $(100 \mu \mathrm{L})$ was dispensed into a pipette-tipbored well in PDA plate that was already inoculated with a mycelial plug of actively growing tested fungus at the center of the medium. Eleven fungal species from the culture collection of PT Wilmar Benih Indonesia ( $G$. boninense B29, Curvularia oryzae, $P$. noxius, Colletotrichum capsici, Neurospora crassa, Pythium acanthicum, Trichoderma asperellum, S. rolfsii, Rhizopus oligosporus, Fusarium oxysporum and Saccharomyces cerevisiae) were used in this experiment. Fungal cultures were incubated at $28 \pm 2{ }^{\circ} \mathrm{C}$ for $6 \pm 2$ days. After the incubation period, inhibition zones $(\mathrm{mm})$ were measured. Antifungal testing against $S$. cerevisiae was done as follows: a single colony of $S$. cerevisiae was suspended in $1 \mathrm{~mL}$ of $0.85 \%(\mathrm{w} / \mathrm{v})$ sterile saline water. Yeast suspension $(100 \mu \mathrm{L})$ was spread on PDA media. PDA was bored using the same methods as previously described, and PHP12 cell-free supernatant $(100 \mu \mathrm{L})$ was added to the well. As a positive control, $100 \mu \mathrm{L}$ of commercial fungicide Dithane ${ }^{\mathrm{TM}}$ Rainshield ${ }^{\mathrm{TM}}$ (containing mancozeb $75 \%$ (w/w); Dow AgroSciences, Australia) was added into the PDA wells. King's B liquid medium was used as a negative control. The antifungal activity of the positive control was also tested against $S$. cerevisiae. The diameter of the growth inhibition zone was measured after incubation at $30^{\circ} \mathrm{C}$ for $48 \mathrm{~h}$. For each antifungal test, e.g. PHP12 cell-free supernatant tested against $G$. boninense, two wells were used in each plate and the plate was duplicated. Thus, for each antifungal test, four wells were used. The mean for each fungal inhibition zone was therefore derived from average of four inhibition zones.

\section{Extraction of antifungal compounds}

The overnight PHP12 culture was centrifuged at $10,000 \times$ $g$ for $10 \mathrm{~min}$ to collect cell-free culture supernatant. Precipitation of the antifungal compounds was carried out by adding ammonium sulfate into the cell-free culture to the concentration of $20 \%(\mathrm{w} / \mathrm{v})$. The mixture was stirred 
on ice for one hour, followed by centrifugation at $10,000 \times$ $g$ for $15 \mathrm{~min}$. The pellet was collected, and ammonium sulfate was added into the supernatant to the concentration of $40 \% \quad(\mathrm{w} / \mathrm{v})$. After stirring and centrifugation, the pellet was collected again, and the supernatant was subjected to another two steps of precipitation with ammonium sulfate at $60 \%$ and $80 \%$ $(w / v)$. Pellets were collected from the four fractions, each resuspended in $35 \%(\mathrm{v} / \mathrm{v})$ acetonitrile and concentrated to 20 times of the original volume. The suspensions were tested for antifungal activity against $S$. cerevisiae.

\section{Reversed-phase-high performance liquid chromatography (RP-HPLC)}

The four fractions obtained from ammonium sulfate precipitation were subjected to separation by RP-HPLC. The suspension was filtered with a syringe using a $0.2 \mu \mathrm{m}$ nylon filter (Millex ${ }^{\circledR}$ Syringe Filters; Millipore). The filtrate was injected into analytical HPLC with ZORBAX 300SB C-18 column, $5 \mu \mathrm{m}, 4.6 \times 150 \mathrm{~mm}$ (Agilent; Santa Clara, CA, USA) at $30^{\circ} \mathrm{C}$ connected to Agilent 1200 Series (Agilent) and diode array detector (DAD) and detected at the wavelength of $220 \mathrm{~nm}$. HPLC analysis was carried out with a gradient of $0.1 \%(\mathrm{v} / \mathrm{v})$ TFA and acetonitrile (9:1) with a flow rate of $0.5 \mathrm{~mL} / \mathrm{min}$. The gradient was as follows: $10 \%$ acetonitrile for $1.6 \times$ column volume, $10-90 \%$ acetonitrile for $5.4 \times$ column volume, $10 \%$ acetonitrile for $2 \times$ column volume. The total run time was $45 \mathrm{~min}$. Subfractions were collected using a fraction collector. The mobile phase of the collected fractions was evaporated using a vacuum evaporator, and the concentrated extract was resuspended in $35 \%(\mathrm{v} / \mathrm{v})$ acetonitrile. The collected sub-fractions were tested for antifungal activity against $S$. cerevisiae.

\section{Liquid chromatography - electrospray ionization/ mass spectrometry (LC-ESI/MS) analysis}

The sub-fractions were analyzed using an Ultimate 3000 UPLC (Thermo Scientific) coupled to a $Q$ Exactive Plus Orbitrap mass spectrometer (Thermo Scientific) equipped with an ESI source. Samples were diluted in acetonitrile $30 \%$ to the concentration of $100 \mathrm{ppm}$. The separation was performed on an analytical Acclaim PepMap300 C18 column, $3 \mu \mathrm{m}, 1 \times 150 \mathrm{~mm}$. Mobile phase A was $0.1 \%$ formic acid in the water, and mobile phase $B$ was $0.1 \%$ formic acid in acetonitrile. The ratio of $A$ and $B$ used for the gradient was: $98: 2(A: B)$ to $60: 40$ over $2 \mathrm{~min}$; to $30: 70$ over $20 \mathrm{~min}$; to $1: 99$ over $1 \mathrm{~min}$, and held at $1: 99$ for $2 \mathrm{~min}$ before re-equilibrating with $98: 2$ for $4 \mathrm{~min}$. The LC flow rate was $50 \mu \mathrm{L} / \mathrm{min}$, the column temperature was $45^{\circ} \mathrm{C}$, and the sample injection volume was $5 \mu \mathrm{L}$. The LC-MS full scan was performed in positive mode from $\mathrm{m} / \mathrm{z} 100$ to 1500 with sheath gas at 60 and aux gas at 10 (arbitrary units), spray voltage at $3.3 \mathrm{kV}$ and capillary temperature at $275^{\circ} \mathrm{C}$. The normalized collision energy was set at 25 .

\section{RESULTS}

\section{PHP12 is a member of the $B$. cepacia complex}

Identification of PHP12 was conducted based on 16s rRNA. The sequence of 16 s rRNA showed that PHP12 is most closely related to $B$. stagnalis with $99 \%$ similarity (Figure 1). The strain is also closely related to other Burkholderia species, namely B. pyrrocinia, B. stabilis, $B$. vietnamensis, and $B$. cepacia. Based on the result, PHP12 can be considered as a member of $B$. cepacia complex (Bcc), a group of Gram-negative bacteria that consist of species closely related to $B$. cepacia.

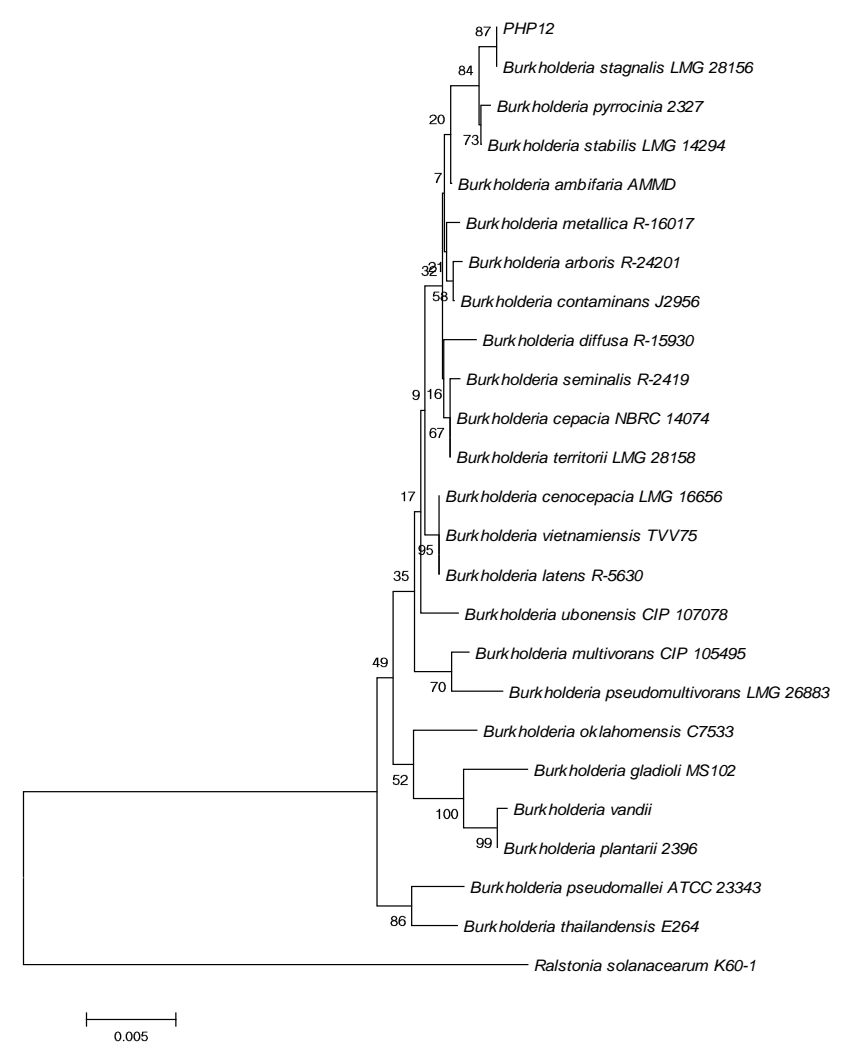

Figure 1: Phylogenetic tree showing the position of PHP12 relative to other Burkholderia species. 16S rRNA sequences from 23 different Burkholderia species were retrieved from National Center for Biotechnology Information (https://www.ncbi.nlm.nih.gov/). Sequences were aligned using ClustalW in Mega X software (Kumar et al., 2018). The phylogenetic tree was constructed using the Neighbor-Joining method. Tree topology was inferred with the Tamura-Nei correction model (Tamura and Nei, 1993) with 1,000 bootstrap replications based on the best substitution model. The tree is drawn to scale, with branch lengths measured in the number of substitutions per site. 

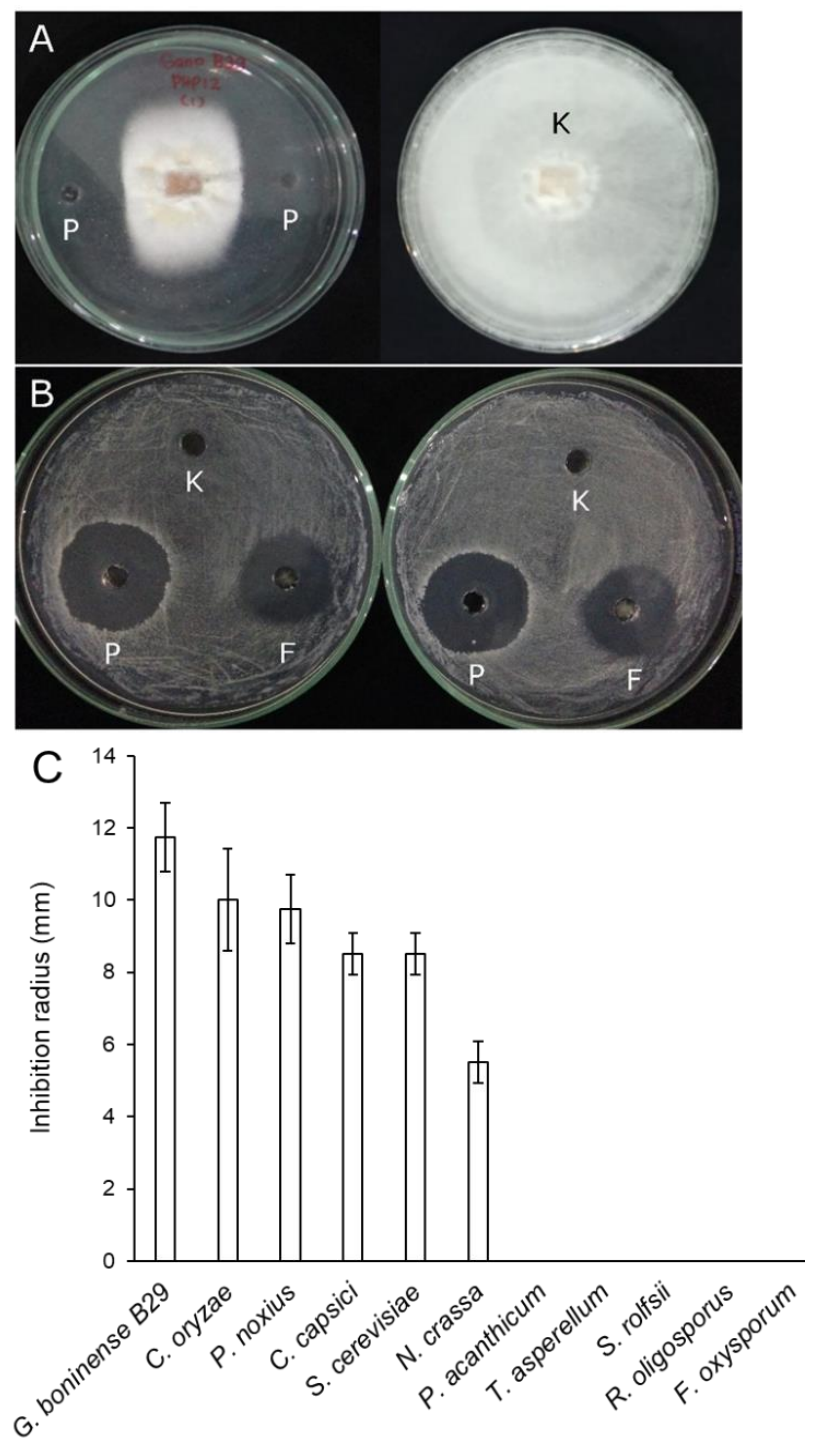

Figure 2: Antifungal assay of PHP12 cell-free culture supernatant against fungi. (A) G. boninense B29 grown on PDA incubated at $28^{\circ} \mathrm{C}$ for $6 \pm 2$ days in the presence (left) and absence (right) of PHP12 cell-free culture supernatant. (B) Antifungal activity of PHP12 cell-free culture supernatant, positive control Mancozeb $0.2 \mathrm{~g} / \mathrm{L}$ (diluted Dithane ${ }^{\circledR}$ M45) and King's B medium as a negative control against $S$. cerevisiae grown on PDA at $30{ }^{\circ} \mathrm{C}$ for $48 \mathrm{~h} . \mathrm{K}=$ negative control; $\mathrm{P}=\mathrm{PHP} 12 ; \mathrm{F}=$ commercial Mancozeb fungicide (positive control). (C) The inhibition zone of PHP12 cell-free culture supernatant against various fungal species. Error bars are standard errors of the means $(n=4)$.

\section{The spectrum of antifungal activity of PHP12}

Boiled PHP12 culture was tested against 11 fungal species to determine the antifungal activity of PHP12. The tested fungal species consisted of model organisms ( $S$. cerevisiae, $R$. oligosporus and $N$. crassa), biocontrol agent ( $T$. asperellum) and plant pathogenic fungi ( $G$. boninense B29, $C$. oryzae, $P$. noxius, $C$. capsici, $P$. acanthicum, $S$. rolfsii and $F$. oxysporum). Cell-free supernatant of PHP12 inhibited the growth of $G$. boninense B29, while there was no inhibition shown by the negative control (King's B medium) (Figure 2A). Cellfree supernatant of PHP12 also inhibited the growth of $S$. cerevisiae as did also a commercial fungicide Mancozeb $0.2 \mathrm{~g} / \mathrm{L}$ (diluted Dithane ${ }^{\circledR} \mathrm{M} 45$ ) as a positive control (Figure 2B). Cell-free supernatant of PHP12 inhibited six fungal species namely $G$. boninense, $C$. oryzae, $P$. noxius, C. capsici, S. cerevisiae, and N. crassa (Figure 2C). Strongest inhibition was observed against $G$. boninense $(11.75 \pm 0.96 \mathrm{~mm})$ followed by the plant pathogens $C$. oryzae $(10 \pm 1.41 \mathrm{~mm}), P$. noxius $(9.75 \pm$ $0.96 \mathrm{~mm})$, and $C$. capsici $(8.5 \pm 0.58 \mathrm{~mm})$. The weakest inhibition of PHP12 supernatant was against $N$. crassa $(5.5 \pm 0.58 \mathrm{~mm})$. The inhibition zone of PHP12 against $S$. cerevisiae was the same as $C$. capsici $(8.5 \pm 0.58 \mathrm{~mm})$. However, five other fungal species including the biocontrol agent $T$. asperellum were not inhibited by the culture supernatant of PHP12 (Figure 2C).

\section{Antifungal activity of PHP12 fractions}

Four fractions obtained from precipitation by ammonium sulfate at several concentrations (20, 40, 60 and $80 \%$; henceforth referred to as F2, F4, F6 and F8) were tested for antifungal activity against $S$. cerevisiae (Figure $3 A$ ). Saccharomyces cerevisiae was used as a model organism due to its fast growth with the antifungal zone being observable in less than $48 \mathrm{~h}$. Fraction F2 showed the highest antifungal activity with inhibition radius at $12.75 \pm 0.5 \mathrm{~mm}$. The antifungal activity of the crude fractions decreased following an increase in ammonium sulfate concentration. Leftover supernatant from ammonium sulfate precipitation was also tested for antifungal activity resulting in a clear zone with radius less than $1 \mathrm{~mm}$ (data not shown). However, the antifungal activity could be due to the high concentration of ammonium sulfate in the supernatant as we had also observed a similar narrow clear zone from sterile King's B medium added with $80 \%$ ammonium sulfate (data not shown).

All of the four fractions were subjected to RP-HPLC. The HPLC chromatogram (Figure 3B) showed that the highest intensity of the peaks was in F2, which eluted at 26 min (labeled as sub-fraction 8). The intensity of the peak decreased following an increase of ammonium sulfate concentration, but it could still be detected in the fraction with $80 \%$ ammonium sulfate. It is indicated that although most of the compounds precipitated at $20 \%$ (w/v) ammonium sulfate concentration, some of the compounds were still remained in the solution. These remaining compounds precipitated in the subsequent precipitation with ammonium sulfate.

About 14 sub-fractions were collected from RP-HPLC from each of the four main fractions (henceforth labelled as SF1 to SF14; labels such as F2-SF8 will refer to a sub- 


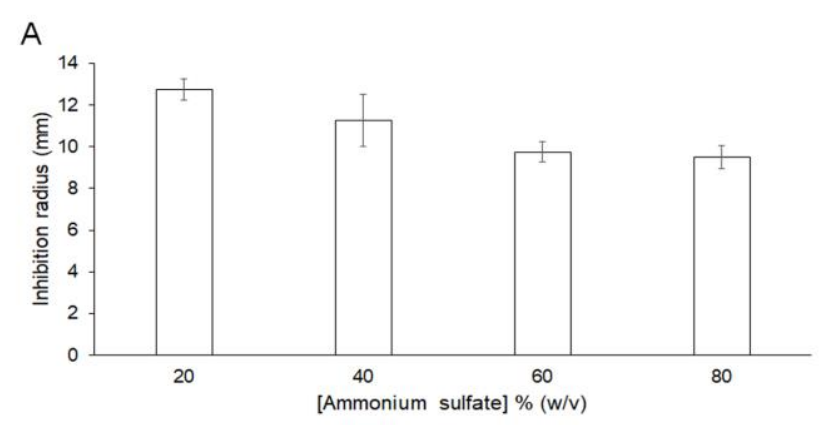

B

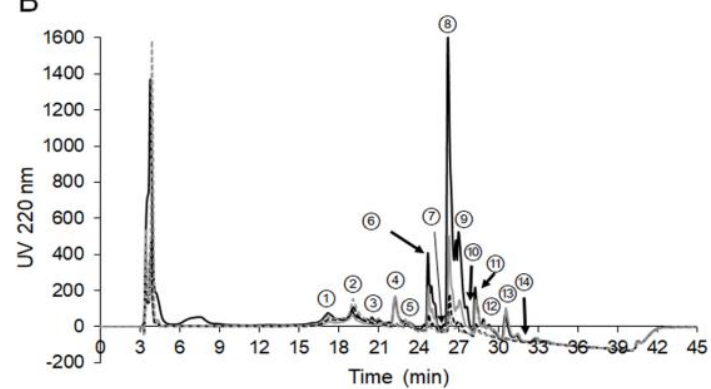

C

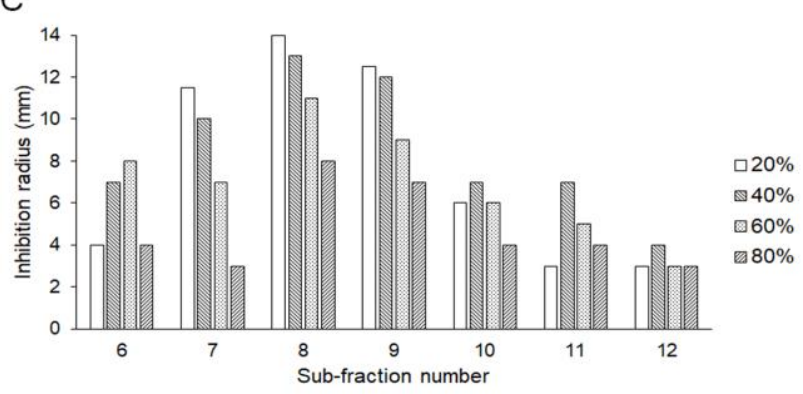

Figure 3: Fractionation of supernatant of PHP12 culture by precipitation with different concentrations of ammonium sulfate $(20,40,60$ and $80 \%(w / v)$; designated as F2, F4, F6 and F8 in the text, respectively). (A) Growth inhibition zone in $S$. cerevisiae by antifungal activity of PHP12 crude ammonium sulfate fractions $(n=4$; error bars are standard errors of the means). (B) RP-HPLC chromatogram of the fractions measured at $220 \mathrm{~nm}$. The circled number refers to the collected sub-fractions. (C). Growth inhibition zone in $S$. cerevisiae by antifungal activity of PHP12 sub-fractions collected from RP-HPLC.

fraction from a specific ammonium sulfate precipitation fraction) resulting in a total of 56 sub-fractions (Figure 3C). All of the 56 sub-fractions were tested for antifungal activity against $S$. cerevisiae, and the result showed that only sub-fractions SF6 to SF12 have antifungal activities. It showed that the antifungal compounds eluted at the same time although they were precipitated at different ammonium sulfate concentrations. SF8 showed the highest antifungal activity than the other sub-fractions (Figure 3C). This trend occurred in SF8 collected from all of the four main fractions (F2 to F8) and indicated that most of the antifungal compounds eluted at $26 \mathrm{~min}$. SF7 to SF9 had a decrease in antifungal activity following an increase in ammonium sulfate concentration. However, the highest antifungal activity in sub-fractions SF10 to SF12 was from the fractions originated from $40 \%$ ammonium sulfate concentration (F4). Some subfractions (Figure $3 \mathrm{C}$ ) had higher antifungal activity when compared to the crude fractions (e.g.: F2-SF8 to F2 and F4-SF8 to F4) (Figure 3A) because the sub-fractions were prepared to be more concentrated (20 times) than the crude fractions. Thus, the antifungal activities of subfractions collected after RP-HPLC could not be compared directly with the crude fractions from ammonium sulfate precipitation.

\section{Identification of PHP12 antifungal compound}

All sub-fractions that showed antifungal activity were subjected to LC-ESI/MS to identify the antifungal compounds of PHP12. Considering that antifungal activities were present in multiple sub-fractions, the target compounds were determined if they were being present conservatively in all sub-fractions and showed different MS intensity in correlation with the antifungal activity of each respective fractions. Based on this hypothesis, a compound with $\mathrm{m} / \mathrm{z} 1211.63$ was suspected as the main antifungal compound of PHP12. Due to protonation of the molecule in MS, the molecular weight was assigned as $1210.63 \mathrm{Da}$ (henceforth the compound is referred to as AF-1210). There were no other molecules in all of the sub-fractions, aside from AF-1210, that fulfilled the previously mentioned condition to be suspected as the target antifungal. MS chromatogram of F2-SF6 and F2SF8 showed the presence of AF-1210 but with different peak intensity (Figure 4A). The peak eluted at $7.51 \mathrm{~min}$ and was also detected in other sub-fractions. Similar to sub-fractions F2-SF6 and F2-SF8, peak intensity in the other fractions also varied in correlation with the antifungal activity of each fraction.

Fragmentation of AF-1210 showed a profile similar to that of an oligopeptide. MS/MS profile also showed that AF-1210 is decorated with a sugar monomer and therefore could be a glycopeptide (Figure 4B). The decorated AF-1210 has the $m / z$ 1373.68, which corresponds to the addition of hexose $(+162 \mathrm{Da})$ and was present in all fractions. Interestingly, F2-SF8 also showed a variation of AF-1210 with the molecular weight of 1349 $\mathrm{Da}$ (not shown). It is possible that this variant was decorated with a pentose instead of hexose. However, this variant was absent in the other sub-fractions.

Identification of the amino acid sequence of AF-1210 was not as successful as the fragmentation. It resulted in the loss of several residues that did not correspond to the molecular weight of conventional amino acids. This suggested that the compound consisted of modified amino acid that makes identification with MS was difficult. So far, we have identified three amino acid residues, namely glutamine, histidine, and tyrosine (Figure 4B). These amino acids were detected as monomers in the MS profile and thus it was not possible to pinpoint their position in the structure. 
A

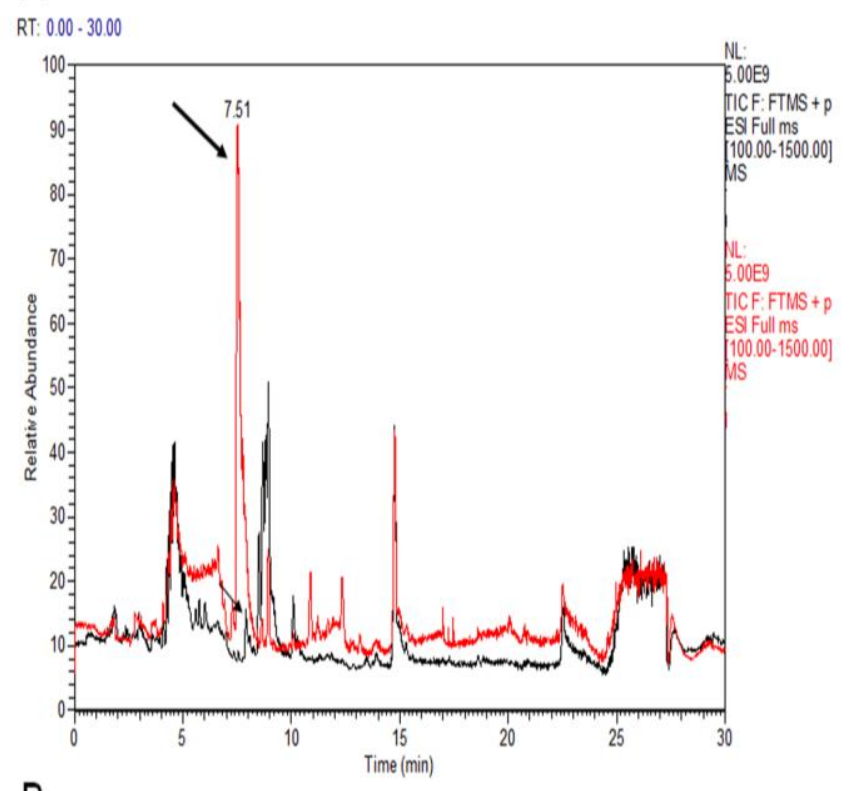

B

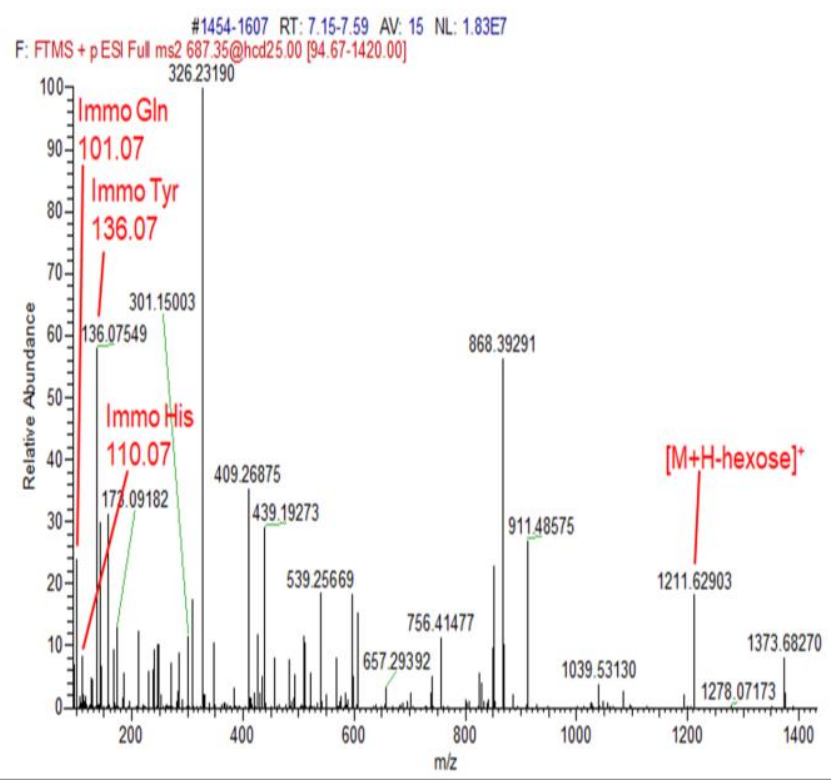

Figure 4: (A) ESI-MS/MS chromatogram of PHP12 subfractions \#6 (black line) and \#8 (red line) from precipitation with ammonium sulfate $20 \% \quad(\mathrm{w} / \mathrm{v})$ (designated as F2-SF6 and F2-SF8 respectively). Black arrow indicates the presence of suspected antifungal compound AF-1210. (B) ESI-MS/MS fragmentation spectrum of parent ion of AF-1210 (m/z 1373.68). Red annotations refer to aglyconated AF-1210 ( $\mathrm{m} / z$ 1211.63) and immonium ions of glycine $(\mathrm{m} / \mathrm{z} 101.07)$, tyrosine $(\mathrm{m} / \mathrm{z}$ 136.07) and histidine ( $\mathrm{m} / \mathrm{z} 110.07)$.

\section{DISCUSSION}

We have characterized a potentially novel antifungal compound that showed strong inhibition against plant pathogenic fungi, particularly $G$. boninense. PHP12 is closely related to $B$. stagnalis. Burkholderia stagnalis is a member of Bcc that has only been recently described (De Smet et al., 2015), therefore reports on its characteristics are limited. To the best of our knowledge, this is the first study to evaluate the antifungal activity of $B$. stagnalis. Members of the Bcc group have been reported to be potential as biocontrol and plant growth promoters in agriculture. However, some strains are also known as human pathogenic bacteria, especially in immunocompromised individuals such as people with cystic fibrosis (CF). In the initial description by De Smet et al. (2015), strains were isolated from soil, tracheal aspiration of non-CF patient and sputum from CF patient. Strain PHP12 used in this study was isolated from soil, and it is unknown whether this strain is pathogenic or non-pathogenic to human. It has been reported that the overlapping taxonomic designation in Burkholderia cannot reflect unambiguously and clearly the division of pathogenic and beneficial strains (De Smet et al., 2015). Pathogenic Bcc strains are distinct from non-pathogenic strains based on fungal-virulence gene loci (Deng et al., 2016). Considering that PHP12 strain will only be applied in agriculture, it is expected not to cause health hazard to humans.

Some species of Burkholderia are well-known as antifungal producers. One of the most well-studied antifungal compounds produced by Burkholderia is pyrrolnitrin, a derivative of tryptophan. Pyrrolnitrin produced by $B$. pyrrocinia has been used to treat plant diseases caused by Rhizoctonia solani (Hill et al., 1994). $B$. contaminans has been reported to produce cyclic oligopeptide, occidiofungin, which was described as decorated with a xylose residue (Lu et al., 2009). Lu et al. (2009) also described the presence of novel amino acids that are linked to an acyl chain in B. contaminans. Other modifications in occidiofungin include the incorporation of $D$-amino butyric acid and $\beta$-hydroxy modification of tyrosine and asparagine ( $\mathrm{Gu}$ et al., 2011). The same modifications could contribute to the difficulty in identifying the amino acid sequence of AF-1210 using MS as they could lead to oligopeptide fragments with molecular mass different from conventional amino acids. The result indicated that the compound AF-1210 from PHP12 showed similar characteristic to that of occidiofungin, i.e., the presence of modified amino acids and sugar residues. However, the molecular weight of AF-1210 (1210 Da) was less than occidiofungin (1215 $\mathrm{Da}$ ). The fragmentation profile of the compound also did not fit with the amino acids that make up the structure of occidiofungin. Based on the MS data, it is possible that AF-1210 to be either a novel compound or a previously undescribed variation of other oligopeptides from Burkholderia. An analysis using NMR is necessary to elucidate the structure of AF-1210. 
It should be noted that there is a possibility of the existence of other antifungal compounds aside from AF1210. As previously mentioned, the leftover supernatant derived from precipitation with ammonium sulfate resulted in a narrow clear zone with radius less than $1 \mathrm{~mm}$. We could not ascertain if the clear zone was a result of fungal inhibition by antifungal molecules or not. However, similarly negligible clear zone was also observed from testing sterile King's B medium that contains $80 \%$ ammonium sulfate indicating that clear zone was due to a high salt content. However, to properly identify the underlying antifungal mechanism of the supernatant, it first needs to be purified, for example, through dialysis. Considering that Burkholderia species are known to produce a wide variety of small antifungal molecules, examination of the leftover supernatant could possibly reveal other antifungal compounds albeit with antifungal activity lower than the AF-1210. However, some molecules, such as pyrrolnitrin, are too small to be precipitated using ammonium sulfate and will be left behind in the supernatant. Therefore, such molecules might have been excluded from our analysis. But given that AF-1210 is definitely the compound showed in MS and has strong antifungal, whereas the possibly antifungal activity of leftover supernatant is negligible, we conclude that AF-1210 is the most compelling candidate for the antifungal compound in PHP12.

Unfortunately, further purification of the antifungal subfraction using HPLC was not successful. An attempt to purify the compound to yield a more definitive data from LC-ESI/MS using longer column and size exclusion chromatography (SEC) was also not successful because the peaks could not be separated. It might be possible to obtain a purer fraction using UPLC equipped with a fraction collector. Further purification using SEC followed by preparative HPLC was proposed by Ross et al. (2014) to purify antifungal polyketides from $B$. gladioli. The same approach could be adapted considering the possibility that similar polyketides might also be produced by PHP12. The method described by Ross et al. (2014) would also allow the fraction to be further characterized using NMR since the method used in our experiment resulted in a fraction that still contained background contaminants which can hinder NMR analysis.

The tolerance of $T$. asperellum towards PHP12 antifungals showed the potential of PHP12 bacterial strain as a candidate for biocontrol agent against plant fungal pathogens. Some members of genus Trichoderma are known to be capable of inhibiting the growth of fungal pathogens such as $G$. boninense and $P$. noxius (Angel et al., 2016; Kwan et al., 2016). Schwarze et al. (2012) reported that Trichoderma sp. can reduce the rate of wood decomposition from white rot disease caused by $P$. noxius. On the other hand, there are also several reports on the ability of bacteria in inhibiting $P$. noxius. Several strains of actinomycetes showed antagonistic activity against $P$. noxius through the production of chitinase (Yanti et al., 2012). In this study, we showed that PHP12 was able to inhibit the growth of $P$. noxius.
Bacterial species such as Burkholderia and Bacillus can inhibit G. boninense (Buana et al., 2014; Pramudito et al., 2018). Moreover, Alexander et al. (2015) stated that Bacillus sp. has synergistic activity with Trichoderma sp. in inhibiting $G$. boninense. The synergism is due to the presence of cell wall degrading enzymes produced by fungi (Trichoderma sp.), which in turn increases the accessibility of bacterial antifungal compounds (Woo et al., 2002). Further research is needed to investigate the possible synergistic mechanism between PHP12 and Trichoderma sp.

\section{CONCLUSION}

In conclusion, this study showed that $B$. stagnalis PHP12 has a potential to be used as a biocontrol agent as it is capable of inhibiting pathogenic fungal strains but not the beneficial ones. The compound was suspected to be a small oligopeptide but further elucidation of its structure was found to be difficult due to modification of the amino acid building blocks. Nevertheless, PHP12 can therefore be used or tested for field application in the plantation industry. We hope that our findings would initiate further research on the antifungal activity of $B$. stagnalis especially on strains related to PHP12.

\section{ACKNOWLEDGEMENTS}

The authors would like to thank the R\&D staff of PT. Wilmar Benih Indonesia, Maria Indah Purnamasari for her help in isolating PHP12 strain.

\section{REFERENCES}

Aderungboye, F. O. (1977). Diseases of the oil palm. Proceedings of the National Academy of Sciences of the United States of America 23(3), 305-326.

Agrios, G. (2005). Plant Pathology. 5th Edn. Academic Press. Cambridge, MA, USA. pp. 1-952.

Alexander, A., Dayou, J. and Chong, K. -P. (2015). Morphological changes of Ganoderma boninense mycelia after challenged by Trichoderma and Bacillus. AIP Conference Proceedings 1699(1), 020075.

Angel, L. P. L., Yusof, M. T., Ismail, I. S., Ping, B. T. Y., Azni, I. N. A. M., Kamarudin, N. H. and Sundram, S. (2016). An in vitro study of the antifungal activity of Trichoderma virens $7 \mathrm{~b}$ and a profile of its non-polar antifungal components released against Ganoderma boninense. Journal of Microbiology 54, 732-744.

Buana, R. F. N., Wahyudi, A. T. and Toruan-Mathius, N. (2014). Control activity of potential antifungalproducing Burkholderia sp. in suppressing Ganoderma boninense growth in oil palm. Asian Journal Agricultural Research 8, 259-268.

Cordova-Kreylos, A. L., Fernandez, L. E., Koivunen, M., Yang, A., Flor-Weiler, L. and Marrone, P. G. (2013). Isolation and characterization of Burkholderia rinojensis sp. nov., a non-Burkholderia cepacia complex soil bacterium with insecticidal and miticidal 
activities. Applied and Environmental Microbiology 79, 7669-7678.

Darma, R., Purnamasari, M. I., Agustina, D., Pramudito, T. E., Sugiharti, M. and Suwanto, A. (2016). A strong antifungal-producing bacteria from bamboo powder for biocontrol of Sclerotium rolfsii in melon (Cucumis melo var. amanta). Journal of Plant Pathology and Microbiology 7, 334.

De Smet, B., Mayo, M., Peeters, C., Zlosnik, J. E. A., Spilker, T., Hird, T. J., LiPuma, J. J., Kidd, T. J., Kaestli, M., Ginther, J. L., Wagner, D. M., Keim, P., Bel, S. C., Jacobs, J. A., Currie, B. J. and Vandamme P. (2015). Burkholderia stagnalis sp. nov. and Burkholderia territorii sp. nov., two novel Burkholderia cepacia complex species from environmental and human sources. International Journal of Systematic and Evolutionary Microbiology 65, 2265-2271.

Deng, P., Wang, X., Baird, S. M., Showmaker, K. C., Smith, L., Peterson, D. G. and Lu, S. (2016). Comparative genome-wide analysis reveals that Burkholderia contaminans MS14 possesses multiple antimicrobial biosynthesis genes but not major genetic loci required for pathogenesis. Microbiology Open 5, 353-369.

Ghisalberti, E. L. and Rowland, C. Y. (2004). Antifungal metabolites from Trichoderma harzianum. Journal of Natural Products 56(10), 1799-1804.

Glick, B. R. and Bashan, Y. (1997). Genetic manipulation of plant growth-promoting bacteria to enhance biocontrol of phytopathogens. Biotechnology Advances 15, 353-378.

Gu, G., Smith, L., Liu, A. and Lu, S. -E. (2011). Genetic and biochemical map for the biosynthesis of occidiofungin, an antifungal produced by Burkholderia contaminans strain MS14. Applied and Environmental Microbiology 77, 6189-6198.

Hill, D. S., Stein, J. I., Torkewitz, N. R., Morse, A. M., Howell, C. R., Pachlatko, J. P., Becker, J. O. and Ligon, J. M. (1994). Cloning of genes involved in the synthesis of pyrrolnitrin from Pseudomonas fluorescens and role of pyrrolnitrin synthesis in biological control of plant disease. Applied and Environmental Microbiology 60, 78-85.

Hwang, J., Chilton, W. S. and Benson, D. M. (2002). Pyrrolnitrin production by Burkholderia cepacia and biocontrol of Rhizoctonia stem rot of poinsettia. Biological Control 25, 56-63.

Katoch, M. and Pull, S. (2017). Endophytic fungi associated with Monarda citriodora, an aromatic and medicinal plant and their biocontrol potential. Pharmaceutical Biology 55, 1528-1535.

Kumar, S., Stecher, G., Li, M., Knyaz, C. and Tamura, K. (2018). MEGA X: Molecular evolutionary genetics analysis across computing platforms. Molecular Biology and Evolution 35, 1547-1549.

Kwan, H. S., Bas, M. C., Schwarze, F. W. M. R., Chu, L. M., Lam, R. Y. C., Tang, A. M. C., Ribera, J., Schubert, M. and Leung, M. W. K. (2016). In-vitro evaluation of antagonistic Trichoderma strains for eradicating Phellinus noxius in colonised wood. Journal of Tropical Forest Science 28, 457-468.

Lu, S. -E., Novak, J., Austin, F. W., Gu, G., Ellis, D., Kirk, M., Wilson-Stanford, S., Tonelli, M. and Smith L. (2009). Occidiofungin, a unique antifungal glycopeptide produced by a strain of Burkholderia contaminans. Biochemistry (Moscow) 48, 8312-8321.

Majeed, R. A., Shahid, A. A., Ashfaq, M., Saleem M. Z. and Haider, M. S. (2015). First report of Curvularia lunata causing brown leaf spots of rice in Punjab, Pakistan. Plant Disease 100(1), 219.

Maznah, Z., Halimah, M., Ismail, S. and Idris, A. S. (2015). Dissipation of the fungicide hexaconazole in oil palm plantation. Environmental Science and Pollution Research 22, 19648-19657.

Omamor, I. B., Asemota, A. O., Eke, C. R. and Eziashi, E. I. (2007). Fungal contaminants of the oil palm tissue culture in Nigerian institute for oil palm research (NIFOR). African Journal of Agricultural Research 2, 534-537.

Pramudito, T. E., Agustina, D., Nguyen, T. K. N. and Suwanto, A. (2018). A novel variant of narrowspectrum antifungal bacterial lipopeptides that strongly inhibit Ganoderma boninense. Probiotics and Antimicrobial Proteins 10, 110-117.

Romero, D., Vicente, A. D., Olmos, J. L., Dávila J. C. and Pérez-García, A. (2007). Effect of lipopeptides of antagonistic strains of Bacillus subtilis on the morphology and ultrastructure of the cucurbit fungal pathogen Podosphaera fusca. Journal of Applied Microbiology 103, 969-976.

Ross, C., Opel, V., Scherlach, K. and Hertweck, C. (2014). Biosynthesis of antifungal and antibacterial polyketides by Burkholderia gladioli in coculture with Rhizopus microsporus. Mycoses 57, 48-55.

Schwarze, F. W. M. R., Jauss, F., Spencer, C., Hallam, C. and Schubert, M. (2012). Evaluation of an antagonistic Trichoderma strain for reducing the rate of wood decomposition by the white rot fungus Phellinus noxius. Biological Control 61, 160-168.

Tamura, K. and Nei, M. (1993). Estimation of the number of nucleotide substitutions in the control region of mitochondrial DNA in humans and chimpanzees. Molecular Biology and Evolution 10, 512-526.

Than, P. P., Prihastuti, H., Phoulivong, S., Taylor, P. W. J. and Hyde, K. D. (2008). Chilli anthracnose disease caused by Colletotrichum species. Journal of Zhejiang University Science B 9, 764-778.

Woo, S., Fogliano, V., Scala, F. and Lorito, M. (2002). Synergism between fungal enzymes and bacterial antibiotics may enhance biocontrol. Antonie Van Leeuwenhoek 81, 353-356.

Yanti, Y., Zainon, M. N. and Marshida, A. H. U. (2012). Antagonistic effects of actinomycetes towards plant pathogen Phellinus noxius. In: 2012 IEEE Colloquium on Humanities, Science and Engineering (CHUSER). 2012 IEEE Colloquium on Humanities, Science and Engineering Research, Magellan Sutera Resort, Kota Kinabalu, Sabah, Malaysia. pp. 419-423. 